\title{
Surveillance Progress for Crucial Vector-Borne Parasitic Diseases in China
}

\author{
Fan Yang ${ }^{1}$; Zhengbin Zhou ${ }^{1}$; Yuan Fang ${ }^{1}$; Xinyu Feng'; Qin Chen ${ }^{1}$; Pin Yang ${ }^{1}$; Jing Xu' ${ }^{1}$; Shizhu Li ${ }^{1, *}$
}

China has achieved significant progress in controlling parasitic diseases through extensive campaigns over the last seven decades. In 2015, the criteria for schistosomiasis transmission control were met nationwide (1), and no indigenous malaria cases have been reported in China since 2017 (2). Moreover, the number of areas with endemic visceral leishmaniasis has been markedly reduced from 16 to 7 provincial-level administrative divisions (PLADs) (3). Surveillance plays an indispensable role in vector-borne parasitic disease control and elimination. Epidemiological data based on surveillance can be used to elucidate disease epidemiology, formulate and adjust control strategies, evaluate intervention effects, and predict epidemic trends.

\section{Prevalence and Trends of Vital Vector-Borne Parasitic Diseases}

Schistosomiasis Presently, integrated surveillance of schistosomiasis in China includes routine and sentinel monitoring, cross-sectional and longitudinal monitoring, and thematic and risk monitoring. Routine and sentinel surveillance of schistosomiasis is based on the National Notifiable Disease Reporting System and extensive sentinel hospitals that mainly monitor the incidence and trends of acute schistosomiasis in endemic areas. To effectively assess the prevalence of schistosomiasis, especially in areas with uncontrolled transmission, the number of acute schistosomiasis cases has been monitored since the early stage of its control. Altogether, 5,651 cases of acute schistosomiasis were reported nationwide between 1999 and 2019, with a peak of 1,114 cases reported in 2003 (4-6). Since then, significant reductions in the number of acute schistosomiasis cases have been observed, with no cases of acute schistosomiasis reported in 2015, 2016, and 2018 (Figure 1A) (4-5).

Cross-sectional and longitudinal monitoring, which consists of multiple rounds of national epidemiological sampling surveys for schistosomiasis, was organized and performed successively in 1989, 1995, and 2004. These three rounds of surveys revealed that the infection rates of humans and livestock showed a steady decrease (Figure 1B); these rates were $10.20 \%$ and $13.29 \%$ in $1989,4.89 \%$ and $9.06 \%$ in 1995 , and $2.50 \%$ and $4.36 \%$ in 2004 , respectively (7).

Repetitive cross-sectional sampling surveys were performed annually in endemic areas of schistosomiasis to observe the prevalence among residents and livestock. The number of schistosomiasis cases increased annually and reached a peak (843,007 cases) in 2003 (5), followed by declines until the lowest historical number of cases $(29,222)$ was reported in 2018 (4) (Figure 1A). The percentage of residents testing positive decreased steadily from $11.10 \%$ $(44,506 / 400,788)$ in 2006 to $0.0015 \%(5 / 327,475)$ in 2019 (4-6) (Figure 1C). Moreover, the percentage of cattle testing positive declined gradually from $4.49 \%$ $(24,863 / 554,003)$ in 2004 to $0.0022 \%(7 / 318,291)$ in 2019 (4-6) (Figure 1C). Although the total snail habitat areas showed some variance recently, the areas with infected snails dropped significantly from $103,651.31 \mathrm{hm}^{2}$ in 2004 to zero in 2014 (4-6) (Figure 1D).

Longitudinal monitoring through fixed sentinel surveillance is conducted yearly to observe trends in disease prevalence and the associated risk factors of populations in different endemic areas. In total, 14 fixed sentinel surveillance sites were established between 1990 and 1998 and gradually expanded to 21 sites between 2000 and 2004, 81 sites between 2005 and 2014, and 458 sites from 2015 onwards to meet the updated requirements for proper schistosomiasis control (7). The infection rate of the inhabitants and livestock decreased from $0.34 \%$ and $0.75 \%$ in 2012 to $0.0015 \%$ and zero in 2018, respectively (4-6).

Malaria In China, malaria is transmitted by Anopheles sinensis, An. anthropophagus, An. mininus, and $A n$. dirus mosquitoes. However, no indigenous malaria cases have been reported in China since 2017. Routine malaria surveillance mainly includes case 

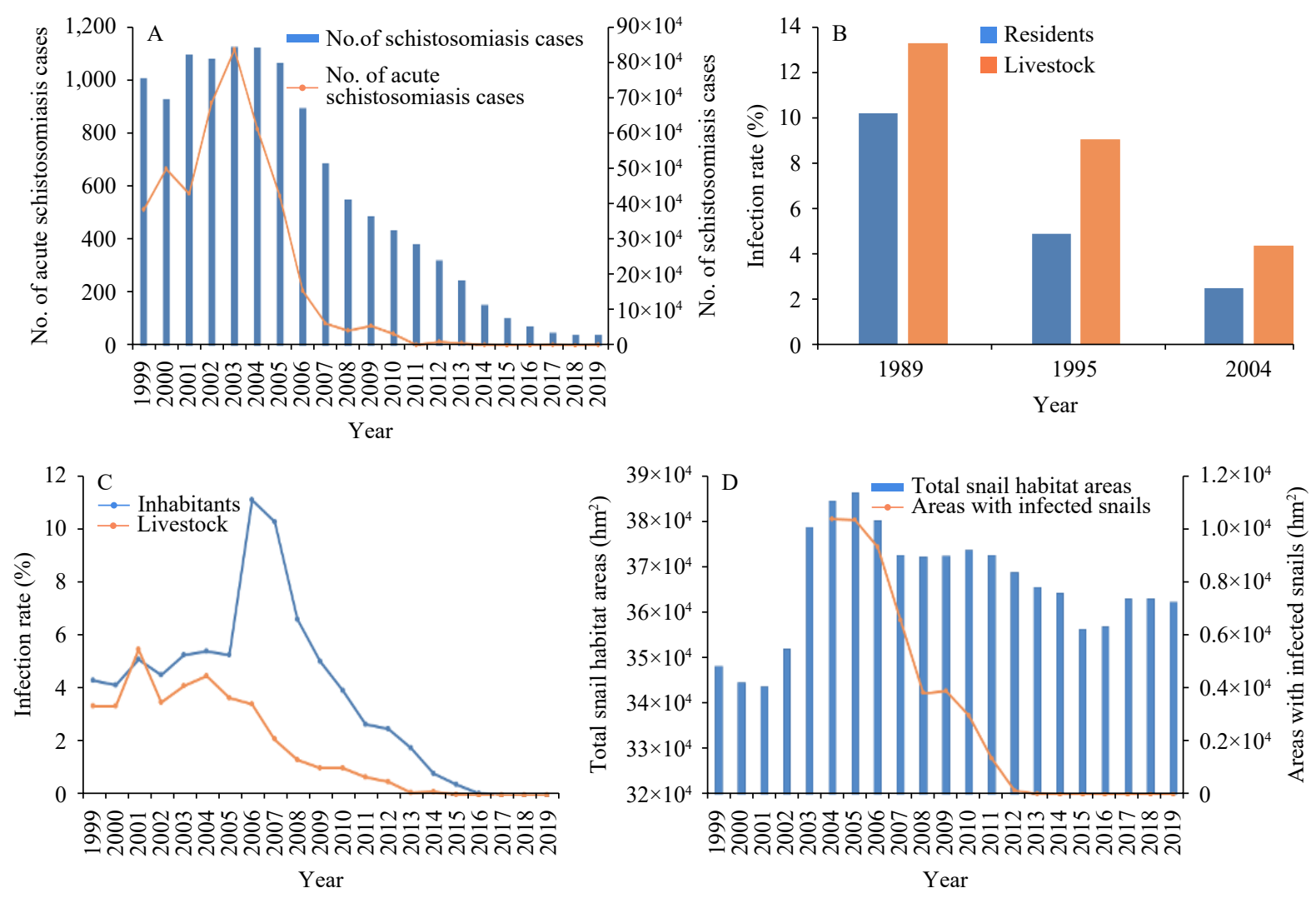

FIGURE 1. Schistosomiasis surveillance results in China, 1999-2019. (A) Number of schistosomiasis cases and acute schistosomiasis cases in China, 1999-2019; (B) National schistosomiasis epidemiological sampling surveys conducted in 1989, 1995, and 2004; (C) Inhabitant and livestock infection rates in schistosomiasis-endemic areas in China, 1999-2019; (D) Total area with snails (1999-2019) and infected snails (2004-2019) in China.

reporting, re-examination, and epidemiological investigation and disposal, as well as blood examinations of patients with fever. The "1-3-7" approach indicates that all malaria cases are to be reported within 1 day ( 24 hours) of diagnosis, epidemiological investigations are to be conducted within 3 days, and foci with cases are to be identified, investigated, and disposed within 7 days (8). In total, 374,127 cases of malaria were reported nationwide between 2000 and 2019, including 46,785 cases of Plasmodium falciparum and 486 deaths (9-11). The number of reported malaria cases declined sharply from 64,178 in 2006 to 4,479 in 2011 and began to level off in 2014; an annual average of 2,983 cases reported between 2014 and 2019 (9-11) (Figure 2A).

Malaria cases imported from abroad have become the primary source of malaria (98\%; 24,304/24,745) since 2012, with an annual average of 3,038 cases reported for the period 2012-2019 (9-11). The number of $P$. vivax cases has been effectively controlled, falling below 300 for the first time in 2019 (11). Approximately 2,068 cases of $P$. falciparum were reported annually, ranging from 765 cases to 2,908 cases between 2012 and 2019 (9-11). The numbers of cases of $P$. malariae and $P$. ovale increased from 51 and 133 in 2013 to 97 and 298 in 2019, respectively, demonstrating significant increases ( $P$. malariae and $P$. ovale cases were unclassified before 2013) (9-11). A total of two cases of $P$. knowlesi were reported. The number of mixed infections has remained relatively stable at approximately 48 cases reported annually (9-11) (Figure 2B).

Visceral Leishmaniasis Visceral leishmaniasis is a disease transmitted by sandflies. Several species of sandflies, including Phlebotomus chinensis, $P$. longiductus, $P$. wui, and $P$. alexandri, are found in China. The incidence of visceral leishmaniasis dropped again in China after strengthened control measures were implemented (Figure 3A). In total, 4,826 visceral leishmaniasis cases were reported in China between 2005 and 2019, including 4,062 cases in endemic areas and 764 cases in non-endemic areas, with an annual average of 322 reported cases (12-13). Of all cases in endemic areas, 659 were anthroponotic-type visceral 

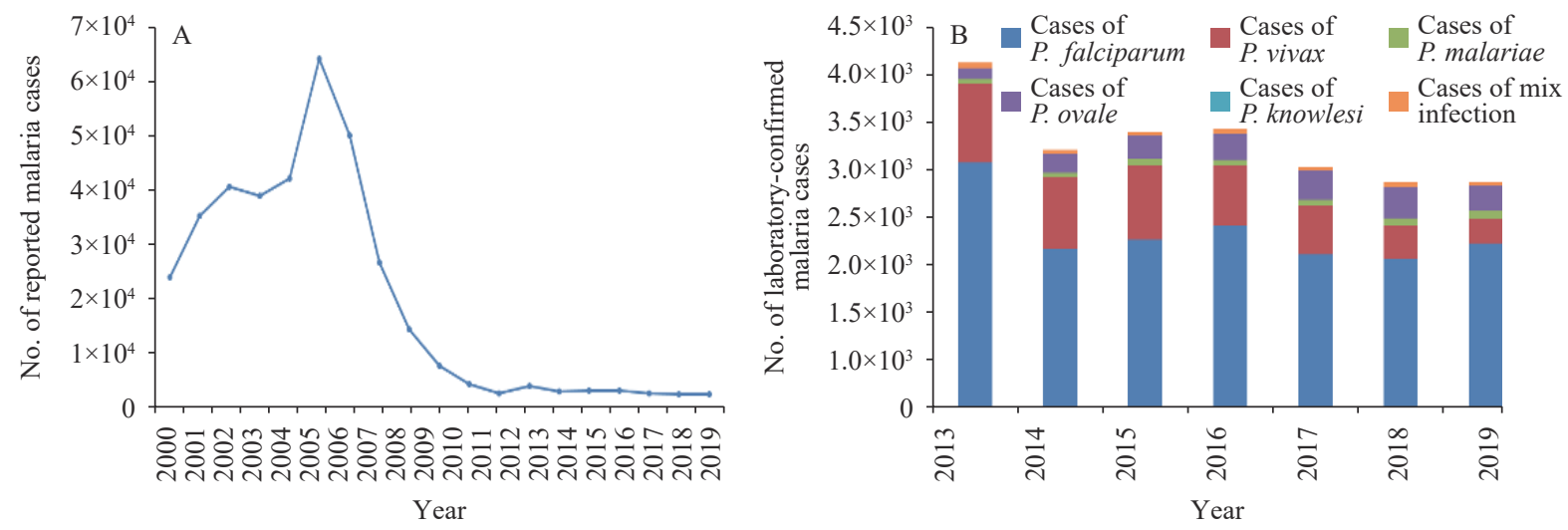

FIGURE 2. Malaria surveillance results in China, 2000-2019. (A) Annual number of reported malaria cases in China, 2000-2019; (B) The number of laboratory-confirmed malaria cases in China, 2013-2019. $P$. malariae and $P$. ovale cases were unclassified before 2013.
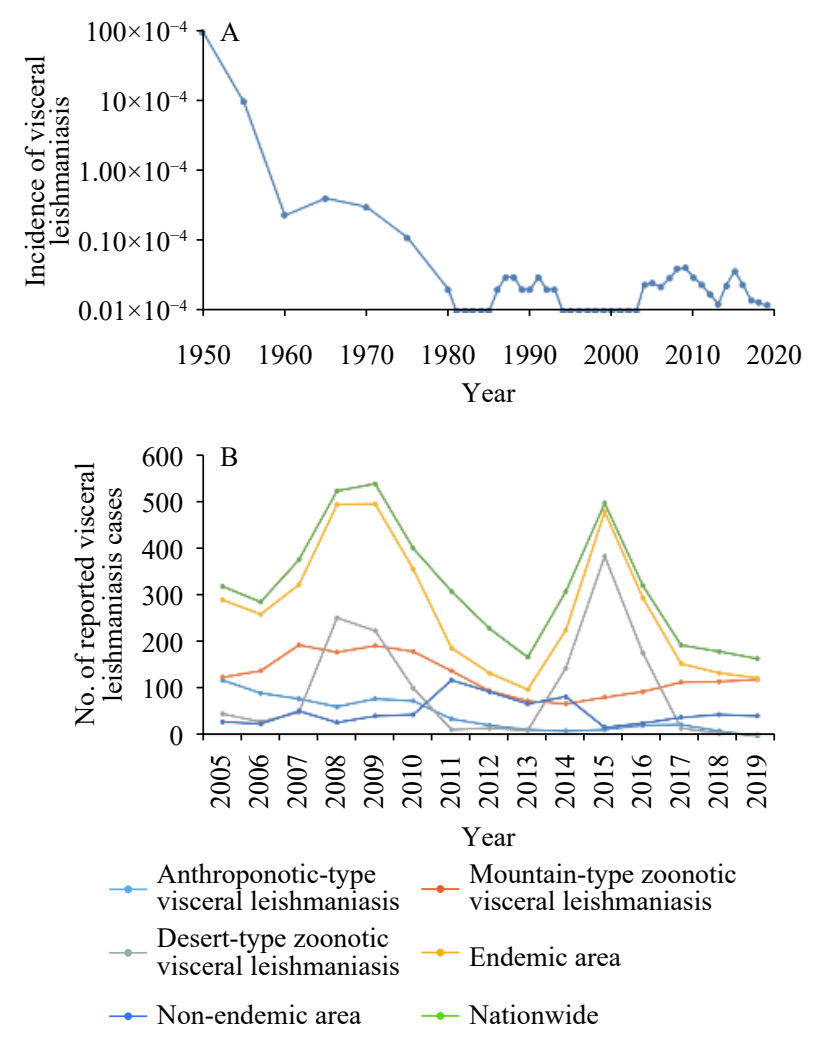

FIGURE 3. Visceral leishmaniasis surveillance results in China, 1950-2019. (A) Incidence of visceral leishmaniasis in China, 1950-2019; (B) The number of reported visceral leishmaniasis cases in China, 2005-2019. Routine surveillance of visceral leishmaniasis based on the National Notifiable Disease Reporting System was established in 2004.

leishmaniasis cases, 1,919 were mountain-type zoonotic visceral leishmaniasis cases, and 1,484 were desert-type zoonotic visceral leishmaniasis cases (12-13). As a consequence of desert-type zoonotic visceral leishmaniasis outbreaks in Jiashi County of Xinjiang Uyghur Autonomous Region, the numbers of reported visceral leishmaniasis cases were 524, 539, and 498 in 2008, 2009, and 2015, respectively (Figure 3B). Cases of visceral leishmaniasis were primarily reported in the PLADs of Xinjiang (2,178 cases), Gansu (1,584 cases), and Sichuan (632 cases), and accounted for approximately $90 \%$ of all cases in China (12).

Emerging, Reemerging, and Imported Vector-Borne Parasitic Diseases The National Survey on the Status of Key Human Parasitic Diseases (2014-2016) indicated that the standardized infection rate of Clonorchis sinensis was $0.25 \%$, which is $24.24 \%$ lower than the standardized infection rate of $0.33 \%$ reported in the first national survey (1988-1992), and 56.89\% lower than the standardized infection rate of $0.58 \%$ reported in the second national survey (2001-2004) (14). Fixed clonorchiasis surveillance sites have been established in the PLADs of Heilongjiang, Jilin, Guangdong, Guangxi, Jiangxi, Guizhou, and Hunan to monitor the infection rate, knowledge, attitude, and behavior of residents as well as the second intermediate host, the reservoir host, and related factors. Partial results indicated that the population of Guangdong displayed a high infection rate of $C$. sinensis (15).

Outbreaks of angiostrongyliasis occurred successively in Wenzhou City of Zhejiang Province (1997), Beijing Municipality (2006), Guangning City of Guangdong Province (2007), and Dali Prefecture of Yunnan Province (2008). The natural epidemic foci of $A$. cantonensis were gradually spreading from the 7 PLADs of Fujian, Guangxi, Guangdong, Hainan, Zhejiang, Jiangxi, and Hunan to other PLADs in China. Surveillance activities to monitor cases, sources of 
infection, and crowd behavior were conducted to prevent recurrent outbreaks of angiostrongyliasis. Conventional tasks included timely diagnosis and treatment of suspected cases in sentinel hospitals, sampling inspections of Pomacea canaliculata snails available in markets and restaurants (16), and a questionnaire survey on dietary knowledge and habits.

No imported cases of Chagas disease have been reported in China thus far. Nonetheless, three imported cases of human African trypanosomiasis have been identified in recent years despite China being a non-endemic country. The first and second imported cases of Trypanosoma brucei gambiense infection in China were reported in 2014 and 2017, respectively, with Gabon being the probable source of infection (17). The first imported case of $T$. b. rhodesiense infection in China was reported in 2017, with Tanzania being the possible source of infection (17).

\section{Surveillance Spotlights and Challenges of Vital Vector-Borne Parasitic Diseases}

Developing precise techniques will be important for schistosomiasis surveillance following continued improvements in disease control. To adapt to a low prevalence of schistosomiasis nationwide, the management of infectious disease sources should be enhanced. In addition to surveillance of schistosomiasis cases and livestock, surveillance of wild animals should be expanded. Meanwhile, monitoring the risk of schistosomiasis ought to be strengthened by improving snail monitoring technology.

Owing to the existence of Anopheles mosquitoes, more emphasis is required on monitoring and managing imported cases to prevent retransmission of malaria, especially at China's borders. Furthermore, mosquito surveillance is crucial for malaria elimination. Although visceral leishmaniasis is sporadically distributed nationwide, it has reemerged in some areas following periods of effective disease control. Hence, sandfly surveillance is essential for the control of visceral leishmaniasis.

Owing to globalization and population mobilization, surveillance of imported vector-borne parasitic diseases should be emphasized. Establishing efficient and sensitive surveillance and early warning response systems is crucial for the prevention and control of vector-borne parasitic diseases. Along with hardware, such as surveillance apparatus and network laboratories, surveillance software and technical schemes should be improved. Furthermore, it is essential to conduct further research on early warning indicators, models of vector-borne parasitic diseases, and the ability of the governing body to deal with public health emergencies and cope with the challenges introduced by globalization and global warming.

Conflicts of interest: There are no conflicts of interest to declare.

doi: $10.46234 /$ ccdcw2020.177

\# Corresponding author: Shizhu Li, lisz@chinacdc.cn.

${ }^{1}$ National Institute of Parasitic Diseases, Chinese Center for Disease
Control and Prevention; Chinese Center for Tropical Diseases
Research; WHO Collaborating Centre for Tropical Diseases; National
Center for International Research on Tropical Diseases, Ministry of
Science and Technology; Key Laboratory of Parasite and Vector
Biology, National Health Commission of China, Shanghai, China.

Submitted: July 14, 2020; Accepted: August 07, 2020

\section{REFERENCES}

1. Zhang LJ, Xu ZM, Qian YJ, Dang H, Lv S, Xu J, et al. Endemic status of schistosomiasis in People's Republic of China in 2015. Chin J Schisto Control 2016;28(6):611 - 7. http://dx.doi.org/10.16250/ j.32.1374.2016246. (In Chinese).

2. Zhang L, Feng J, Zhang SS, Xia ZG, Zhou SS. The progress of national malaria elimination and epidemiological characteristics of malaria in China in 2017. Chin J Parasitol Parasit Dis 2018; 36(3): 201-9. http://d.wanfangdata.com.cn/periodical/zgjscxyjscbzz201803001. (In Chinese).

3. Lun ZR, Wu MS, Chen YF, Wang JY, Zhou XN, Liao LF, et al. Visceral leishmaniasis in China: an endemic disease under control. Clin Microbiol Rev 2015;28(4):987 - 1004. http://dx.doi.org/10.1128/ CMR.00080-14.

4. Zhang LJ, Xu ZM, Guo JY, Dai SM, Dang H, Lv S, et al. Endemic status of schistosomiasis in People's Republic of China in 2018. Chin J Schisto Control 2019;31(6):576 - 82. http://dx.doi.org/10.16250/ j.32.1374.2019247. (In Chinese).

5. Zhang LJ, Dai SM, Xue JB, Li YL, Lv S, Xu J, et al. The epidemiological status of schistosomiasis in P.R. China after the World Bank Loan Project, 2002-2017. Acta Trop 2019;195:135-41. http://dx.doi.org/10.1016/j.actatropica.2019.04.030.

6. Chen XY, Jiang QW, Wang LY, Zhao GM, Zhao Q, Gu YA, et al. Schistosomiasis situation in the People's Republic of China in 2001. Chin J Schisto Control 2002;14(4):241 - 3. http://dx.doi.org/10.16250/ j.32.1374.2002.04.001. (In Chinese).

7. Zhang LJ, Li SZ, Wen LY, Lin DD, Abe EM, Zhu R, et al. The establishment and function of schistosomiasis surveillance system towards elimination in the People's Republic of China. Adv Parasitol 2016;92:117 - 41. http://dx.doi.org/10.1016/bs.apar.2016.03.003.

8. Feng XY, Xia ZG, Vong S, Yang WZ, Zhou SS. Surveillance and response to drive the national malaria elimination program. Adv Parasitol 2014;86:82 - 108. http://dx.doi.org/10.1016/B978-0-12800869-0.00004-4.

9. Feng J, Zhang L, Huang F, Yin JH, Tu H, Xia ZG, et al. Ready for malaria elimination: zero indigenous case reported in the People's Republic of China. Malar J 2018;17:315. http://dx.doi.org/10.1186/ s12936-018-2444-9.

10. Zhang L, Feng J, Zhang SS, Xia ZG, Zhou SS. Epidemiological characteristics of malaria and the progress towards its elimination in China in 2018. Chin J Parasitol Parasit Dis 2019;37(3):241 - 7. 
http://dx.doi.org/10.12140/j.issn.1000-7423.2019.03.001. (In Chinese).

11. Zhang L, Feng J, Xia ZG, Zhou SS. Epidemiological characteristics of malaria and progress on its elimination in China in 2019. Chin J Parasitol Parasit Dis 2020;38(2):133 - 8. http://dx.doi.org/10.12140/ j.issn.1000-7423.2020.02.001. (In Chinese).

12. Zheng CJ, Xue CZ, Wu WP, Zhou XN. Epidemiological characteristics of Kala-azar disease in China, during 2005-2015. Chin J Epidemiol 2017;38(4):431 - 4. http://dx.doi.org/10.3760/cma.j.issn.0254-6450. 2017.04.004. (In Chinese).

13. Zhou ZB, Li YY, Zhang Y, Li SZ. Prevalence of visceral leishmaniasis in China during 2015-2018. Chin J Parasitol Parasit Dis 2020;38(3): 339 - 45 . http://dx.doi.org/10.12140/j.issn.1000-7423.2020.03.013. (In Chinese).

14. Chen YD, Zhou CH, Zhu HH, Huang JL, Duan L, Zhu TJ, et al. National survey on the current status of important human parasitic diseases in China in 2015. Chin J Parasitol Parasit Dis 2020;38(1): 5 - 12. http://dx.doi.org/10.12140/j.issn.1000-7423.2020.01.002. (In Chinese).

15. Deng ZH, Fang YY. Epidemic situation and prevention and control strategy of clonorchiasis in Guangdong Province, China. Chin J Schisto Control 2016;28(3):229 - 33. http://dx.doi.org/10.16250/j.32.1374. 2016081. (In Chinese).

16. Li LS, Zhang RY, Fang YY, Ouyang R, Xie HG, Jiang DW, et al. Prevalence of Angiostrongylus cantonensis infection in snails for sale in Fuzhou and Xiamen. Chin J Parasitol Parasit Dis 2013; 31(6): 464-6. http://d.wanfangdata.com.cn/periodical/zgjscxyjscbzz201306011. (In Chinese).

17. Zhou XN, Qian MB, Priotto G, Franco JR, Guo JG. Tackling imported tropical diseases in China. Emerg Microbes Infect 2018;7(1):12. http://dx.doi.org/10.1038/s41426-018-0022-4. 